\title{
Cambios fonológicos vs obsolescencia lingüística: ¿Cuál es su impacto en la revitalización del rama?
}

\author{
Bénédicte Pivot y Natacha Chevrier \\ Universidad de Lyon \\ (Laboratorio de Dinámica del Lenguaje)
}

Este artículo presenta una mirada cruzada entre los cambios fonológicos, debidos al fenómeno de obsolescencia ${ }^{1}$ lingüística, y el impacto de esos cambios sobre los esfuerzos de revitalización de la lengua. El trabajo está basado en un estudio de caso del idioma rama el cual se consideraba casi extinto en los años ochenta y que desde entonces ha sido beneficiado de un programa de descripción, documentación y revitalización: el Proyecto del Idioma Rama (RLP - Rama Language Project) ${ }^{2}$. El análisis propuesto implica al mismo tiempo el campo de la fonología y el de la sociolingüística de las Lenguas en Peligro (LEP). Se fundamenta en dos encuestas de terreno efectuadas en 2010 y $2011^{3}$.

Después de una presentación de la situación sociolingüística del rama y del contexto en el cual se inscribe el proyecto de revitalización, el fenómeno de obsolescencia lingüística será descrito a través del estudio de la pérdida de un fonema, la nasal velar / ๆ /. Finalmente será analizado el reto que representa para los esfuerzos de revitalización la emergencia de nuevas 'variantes' debidas a este tipo de fenómeno de obsolescencia.

\section{EL RAMA (LEP), OBJETO DE DESCRIPCIÓN- DOCUMENTACIÓN-REVITALIZACIÓN}

La lengua rama de Nicaragua forma parte de la familia de lenguas chibchas, habladas desde Honduras al norte, hasta Venezuela al sur. Es la lengua del grupo del mismo nombre, que cuenta con alrededor de 3,000 miembros que viven en un vasto territorio situado en la Región Autónoma del Atlántico Sur (RAAS) de Nicaragua.

\section{Repartición geográfica de los ramas}

Los dos tercios de los ramas viven en la isla lagunera de Rama Cay, a una decena de kilómetros al sur de la capital regional, Bluefields. Es la comunidad principal con la iglesia y las escuelas primaria y secundaria en las cuales se imparte cursos de rama. Los otros ramas se distribuyen en ocho pequeños grupos de una o varias decenas de personas en el bosque continental. Solo las comunidades más pobladas tienen una escuela primaria ${ }^{4}$.

1 Término utilizado en los trabajos sobre las lenguas en peligro, para designar las evoluciones lingüísticas que ocurren en esas lenguas (N. Dorian (éd.), Investigating obsolescence. Studies in language contraction and death, Cambridge: CambridgeUniversity Press, 1989).

2 Ver <http://www.ddl.ish-lyon.cnrs.fr/aalled/Telechargeable/Nicaragua-final.2.pdf>. Para una presentación del RLP ver entre otros C. Craig, «Language Shift and Language Death: The case of Rama in Nicaragua », International Journal of theSociology of Language 93, Berlin/New-York : Mouton de Gruyter, 1993 ; C. Grinevald, «Quarante ans de perspective sur deux langues en danger : le jakaltek popti’ du Guatemala et le rama du Nicaragua », en C. Grinevald \& M. Bert (dir.),Linguistique de terrain sur langues en danger, Faits de langues 35-36, Paris : Ophrys, 2010 ; C. Grinevald, « Linguistique de terrain sur deux langues en danger : locuteurs et méthodes », en C. Grinevald \& M. Bert (dir.), op. cit. [Grinevald publicó bajo el nombre de Caig hasta 1996].

32010 : seis semanas; Bluefields y Rama Cay ; entrevistas con 19 ramas involucrados en el RLP (adultos principiantes, miembros del gobierno local, niños y jóvenes adultos) (B. Pivot, Evaluation d'une situation d'une langue en danger, lecas du rama au Nicaragua, Université Lyon 2 : memoria de máster 2 : SDL, 2010) / 2011 : 6 semainas ; Bluefields, Rama Cay y dos comunidades ramas de la jungla ; entrevistas y elicitaciones lingüísticas con 12 ramas de tres perfiles lingüísticos diferentes (N. Chevrier, Evolution phonologique d'une langue en danger : le cas du rama du Nicaragua, Université Lyon 2 :memoria de master 2 : SDL, 2011).

4 G. Riverstone, La tierra de nuestros ancestros. Territorio Rama y Creole en el Caribe Nicaragüense, Managua, Nicaragua : IBIS, 2008. 
La repartición geográfica de la población corresponde a la repartición de los locutores de la lengua étnica. En efecto, una migración lingüística hacia la lengua vehicular de la población dominante en la zona -un criollo de base inglesa conocido como el criollo de la costa miskita (Miskitu Coast Creole-MCC) $)^{5}$ - comenzó a desarrollarse al principio del siglo XX. Sin embargo, ésta no fue tan radical en las tierras continentales como en la isla de Rama Cay. De esta forma, si la isla cuenta hoy con un solo locutor tradicional ${ }^{6}$, los cerca de 60 otros hablantes o semi-hablantes ${ }^{7}$ conocidos están repartidos en las otras comunidades.

\section{El rama: lengua en peligro extremo}

El fenómeno de obsolescencia ha sido por largo tiempo apartado por los estudios lingüísticos. Se ha considerado -y desgraciadamente todavía a veces se considera- que una lengua en situación de obsolescencia era una forma degenerada o corrompida, de la lengua original, y que solo la lengua en su estado 'puro’ era digna de interés científico. Ahora se reconoce que ese fenómeno es interesante en sí y que puede revelar situaciones de lenguas en contacto ${ }^{8}$.

El rama es considerado como una "lengua en situación crítica”, en el atlas de las lenguas en peligro de la UNESCO $^{9}$. Esta clasificación se apoya sobre los criterios de vitalidad desarrollados por la UNESCO, como el número de hablantes tradicionales, el estado de la transmisión entre generaciones y la actitud hacia la lengua ${ }^{10}$. En el caso del rama, en 2010, se contaban alrededor de 30 hablantes tradicionales, un número similar de semi-hablantes y una gran mayoría de "neohablantes"11. La lengua sigue el esquema de la "muerte gradual” (gradual death) ${ }^{12}$ por la detención progresiva de la transmisión del rama a los niños. En lo que concierne a la actitud hacia la lengua étnica, ésta ha evolucionado mucho en los últimos 25 años, pasando de un estatus de “tiger language" 13 , lengua de los salvajes de la jungla, al de "nuestra lengua"14, lengua de alto valor simbólico de identificación y de pertenencia étnica (ver sección 3).

En el contexto de las negociaciones por la autonomía de la región atlántica del país y el reconocimiento de los derechos indígenas, el jefe de la comunidad rama solicitó en 1984 al Gobierno Popular Sandinista ayuda para salvar la lengua étnica ${ }^{15}$. Así comenzó el RLP, dirigido por la lingüista Colette Grinevald (Craig en la época).

\section{El proyecto de descripción, documentación/archivo y revitalización de la lengua rama}

Este proyecto se desarrolló en dos fases distintas. En un primer tiempo (1985-1993) tuvo por objetivo lingüístico realizar la primera descripción gramatical de la lengua, sobre la base de diversos textos referentes a la cultura étnica, y la preparación de un diccionario. Al mismo tiempo, el proyecto sostuvo los primeros esfuerzos de revitalización que fueron concebidos y realizados principalmente por una semi-hablante, sobre todo con niños de la isla escolarizados en escuela maternal.

5 J. A. Holm, « Nicaragua's Miskito Coast Creole English », dans J. A. Holm (éd.), Central American English. Varieties ofEnglish Around the World vol. 2, Heidelberg : J.Groos, 1983

6 Es decir, cuya lengua materna es el rama (M. Bert, C. Grinevald, « Proposition de typologie des locuteurs de LED », dans C. Grinevald \& M. Bert (dir.), op. cit., p. 125-126).

7 Hablantes que tienen un dominio parcial del idioma (Ibid., p. 126)

$8 \quad$ N. Dorian (éd.), Investigating obsolescence, op. cit., p. 2-3.

$9 \quad$ C. Moseley (éd.), Atlas des langues en danger dans le monde 3e édition, Paris : Editions UNESCO, 2010. Versión en línea:<http://www.unesco. org/culture/en/endangeredlanguages/atlas>

10 Ver C. Grinevald, J. Costa, «Langues en danger : le phénomène et la réponse des linguistes », dans C. Grinevald \& M.Bert (dir.), op. cit., p. 30

11 J. Costa, « Des derniers locuteurs aux néo-locuteurs : revitalisation linguistique en Europe », dans C. Grinevald \& M. Bert (dir.), op. cit. Los neo-locutores son personas que han aprendido la lengua en contextos de revitalización

12 La situación más frecuentemente señalada en la literatura sobre las lenguas en peligro y en el seno de la cual hay más riesgos de constatar cambios lingüísticos (L. Campbell, M. C. Muntzel, « The structural consequences of language death», dans N. Dorian(éd.), op. cit., p. 184-185; C. Craig, « Language Contact and Language Degeneration », dans F. Coulmas (éd.), TheHandbook of Sociolinguistics, Oxford : Blackwell, 1997, p. 259)

13 C. Grinevald, «Why the Tiger language and not Rama Cay Creole? Language revitalization made harder », en P. K.Austin (éd.), Language Documentation and Description 3, London : HRELP SOAS, 2005

14 Pivot B., op. cit., p. 107.

15 C. Craig, «A constitutional response to language endangerment: the case of Nicaragua », Language 68/1, 1992. 


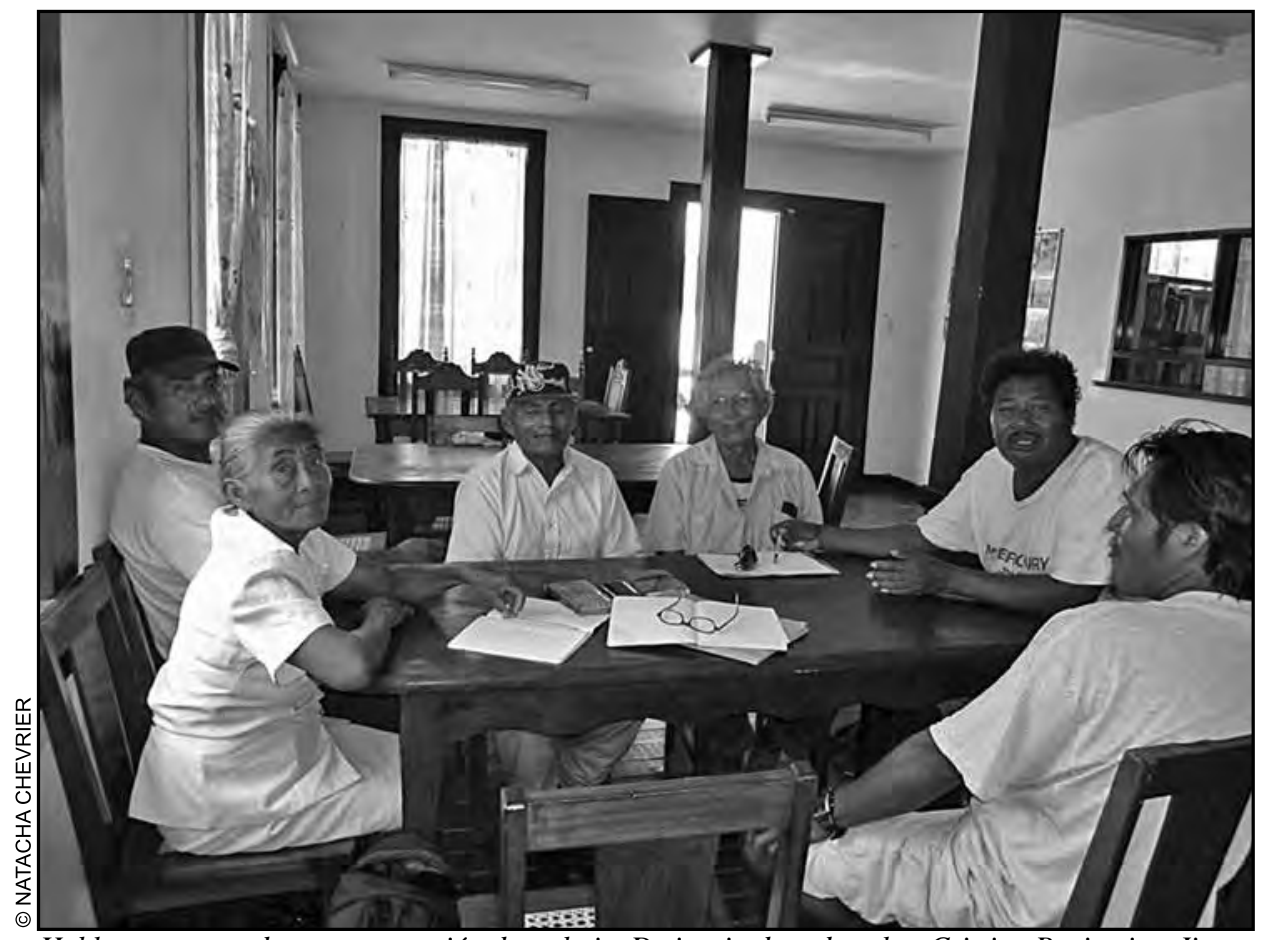

Hablantes ramas durante una sesión de trabajo. De izquierda a derecha: Cristina Benjamins, Jimmy McCrea, Pedro McCrea, Walter Ortiz, Francisco Walter, José Wilson. BICU-CIDCA, Bluefields, 2011.

lo que ella sabía de la lengua y sin la cual no hubiera sido posible jamás el proyecto del idioma rama. Después, las condiciones de terreno se mejoraron, dos hablantes tradicionales se unieron a ellas: Trad.K ${ }^{19}$ (nuera de Miss Nora, que vivía en una comunidad de la selva) y Trad.W (único hablante tradicional que sabía leer y escribir (en español), que vivía en Rama Cay).

\section{Los locutores emergentes:}

Como es frecuente en las situaciones de lenguas en peligro, otros perfiles de hablantes aparecieron en el panorama en función de las actividades y oportunidades ligadas al contexto de revitalización: ${ }^{20}$

Semi-hablantes, como los hijos de Miss Nora (Sem.P) que tienen un conocimiento más limitado de

Desde el 2004, la segunda fase del proyecto se ha orientado hacia el archivamiento ${ }^{16}$ de la documentación del material colectado y hacia acciones de revitalización sostenidas por el Gobierno Territorial Rama-Kriol (GTR-K). Los talleres sobre la lengua organizados y dirigidos por los lingüistas han visto una participación cada vez más grande de hablantes y miembros de la comunidad interesados y dispuestos a integrarse en el proyecto.

\section{Los hablantes del Proyecto Rama ${ }^{17}$}

El proyecto del idioma rama, por la naturaleza misma de proyecto de descripción de una lengua en peligro, se efectuó con la participación de hablantes de varios perfiles distintos. $^{18}$

\section{Un primer equipo de tres hablantes:}

En 1985, C. Craig trabajó principalmente con una semihablante: Miss Nora, que estaba tratando de transmitir la lengua y que se invierten cada vez más desde la muerte de su madre.

Neo-hablantes que fueron alumnos de Miss Nora y después de Trad.W. Sus competencias varían mucho de un individuo al otro y se resumen con frecuencia al conocimiento de listas temáticas de palabras. A propósito de ese perfil de locutores, notemos que “A nivel lingüístico, la variedad que hablan, en general en contextos limitados, es frecuentemente una variedad estandarizada que puede presentar signos importantes de obsolescencia lingüística”21.

Neo-hablantes 'principiantes', que distinguimos como un perfil particular por el hecho que están en fase de aprendizaje voluntario de la lengua. Son un poco mayores para haber sido alumnos de Miss Nora y por eso no han tenido nunca una transmisión directa de la lengua. Sin embargo son los actuales

16 Ver los archivos AILLA : <http://www.ailla.utexas.org/search/view_resource.html?lg_id=67>

17 Este artículo está dedicado a la memoria de Jimmy McCrea, fallecido en abril 2012. Era nieto de Miss Nora e hijo de Pedro McCrea y Cristina Benjamins, miembros principales, con Walter Ortiz, del Proyecto de Lengua Rama (véase Grinevald y Kauffmann, 2004, "Meditaciones en la sombra”, Wani 38). Participó en el presente estudio fonológico, durante varias semanas de trabajo en BICU-CIDCA, de marzo a abril del 2011. Para la temática de la diversidad de hablantes de lenguas en peligro, ver M. Bert, C. Grinevald, op. cit.

19 Los hablantes con quienes los autores trabajaron serán designados por seudónimos : l'abreviación 'Trad.' (tradicional), 'Semi.' (semihablante) o 'App.' (aprendiz = principiante) seguido de l'inicial del nombre..

20 J. Costa, op. cit

$21 \quad$ M. Bert, C. Grinevald, op. cit., p. 129. 


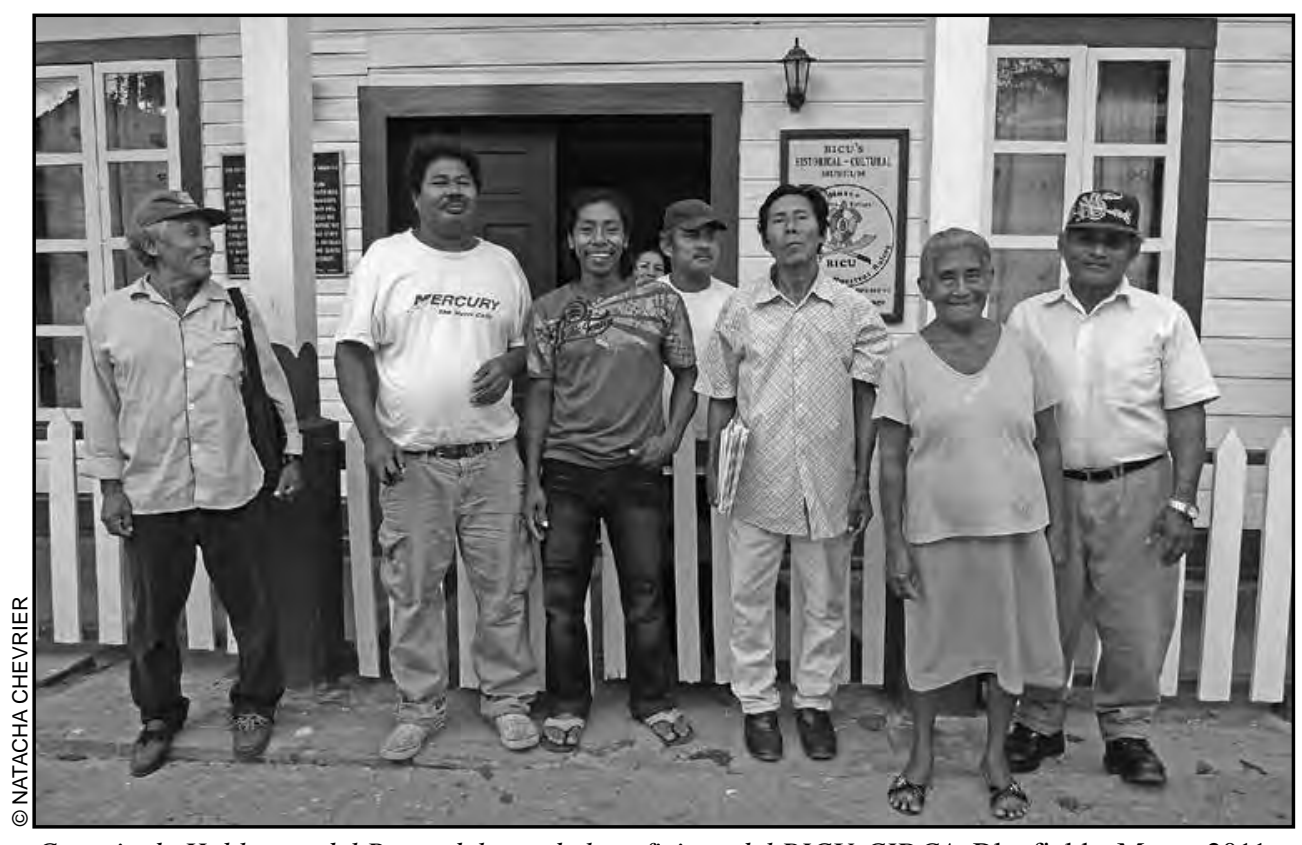

Consejo de Hablantes del Rama delante de las oficinas del BICU-CIDCA, Bluefields, Marzo 2011.

transmisores del rama en las diferentes escuelas de las comunidades (Apr.J. y Apr.S, al igual que dos o tres colegas más).

Nuestro estudio concierne más particularmente a estos dos últimos tipos de perfil.

El estudio fonológico propuesto fue realizado en el campo, con una muestra de estos diferentes tipos de hablantes: cuatro hablantes tradicionales, cinco semi-hablantes y tres neo-hablantes principiantes ${ }^{22}$.

\section{LA PÉRDIDA PROGRESIVA DE LA NASAL VELAR EN LA LENGUA RAMA}

A continuación el análisis detallado de un fenómeno de desaparición fonológica debido a la obsolescencia de la lengua.

\section{[n] en el sistema fonológico del rama.}

Las consonantes fonológicas del rama son las siguientes:

\begin{tabular}{|c|c|c|c|c|c|c|c|c|c|}
\hline & Bilabiales & $\begin{array}{l}\text { Labio- } \\
\text { dentales }\end{array}$ & Alveolares & $\begin{array}{c}\text { Post- } \\
\text { alveolares }\end{array}$ & Palatales & & & $\begin{array}{l}\text { Labio- } \\
\text { velares }\end{array}$ & Glotales \\
\hline Oclusivas & $p \quad b$ & & $d$ & & & $\mathrm{k}$ & 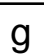 & $\mathrm{kw}$ & \\
\hline Nasales & $\mathrm{m}$ & & $\mathrm{n}$ & & & & $\eta$ & nw & \\
\hline Vibrantes & & & $r$ & & & & & & \\
\hline Fricativas & & (f) & $\mathrm{s} \quad(\mathrm{z})$ & $\int$ & & & & & $\mathrm{h}$ \\
\hline Africadas & & & & $\left(\mathrm{t} \int\right)$ & & & & & \\
\hline Laterales & & & I & & & & & & \\
\hline $\begin{array}{l}\text { Semicon- } \\
\text { sonantes }\end{array}$ & & & & & $\mathrm{j}$ & & & W & \\
\hline
\end{tabular}

Tabla 1. Inventario fonológico del rama ${ }^{23}$

Trad.W, Trad.K, Trad.A, Trad.P ; Sem.P, Sem.J, Sem.R, Sem.W, Sem.L ; App.J, App.S et App.D.

23 Realizado a partir de C. Craig, A Grammar of Rama (Report to National Science Foundation BNS 8511156), 1988, p. 39-41 ; y de C. Grinevald \& al., Turkulka: diccionario de lengua rama, 2002-2006. En línea : <www.turkulka.net> 


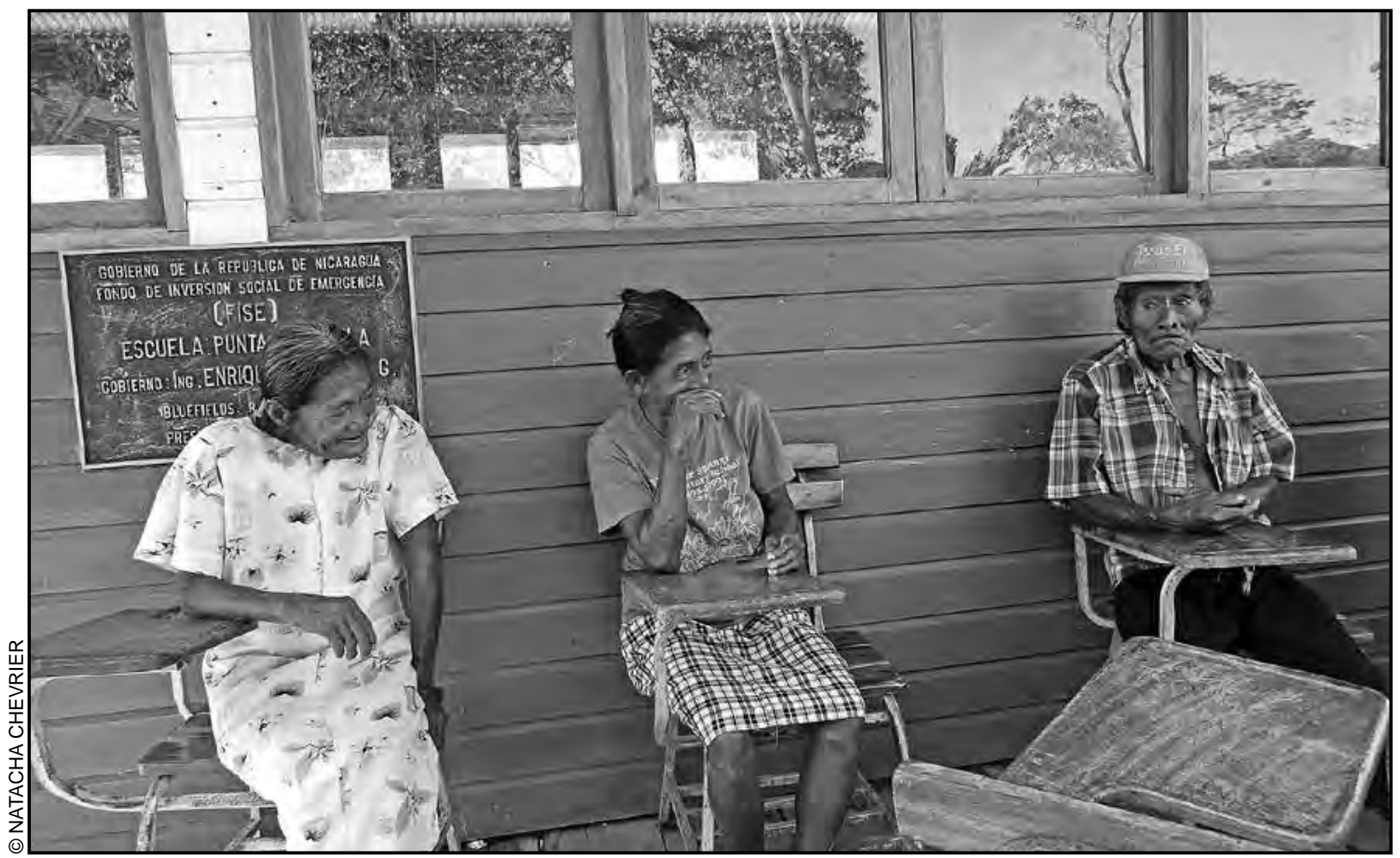

Sesión de trabajo con hablantes en Bangkukuk Taik, Marzo 2011.

La nasal velar es un fonema bastante raro en las lenguas del mundo, pero es muy común en el rama y puede aparecer en todas las posiciones:

Inicial
(1) /nauknauk/ 'araignée'
(2) / puu/ 'maison'
(3) / ’aarak/ 'beaucoup'

Médiana

(4) /narnarinBa/24 'vert'

(5) $/$ niSniS/25 'criquet'

(6) /parna/ 'noir'
Final

(7) / naan / 'lit'

(8) /ulinulin/ 'singe hurleur'

(9) /kiin/ 'tête'

\section{Trabajo de terreno con diferentes perfiles de hablantes}

El trabajo de terreno llevado a cabo con 12 hablantes de perfiles diferentes consistió en la elicitación de una lista de 96 entradas, creadas a partir del diccionario Turkulka ${ }^{26}$ y de elicitación libres.

Con el fin de observar los cambios lingüísticos atestiguados en una variedad más reciente de la lengua, el análisis de la pérdida de la nasal velar se basa esencialmente en los datos cuya fuente son los ocho hablantes no tradicionales.

\subsection{Un cambio bajo el signo de la variación.}

El fenómeno de pérdida de la nasal velar es más complejo que una simple elisión.
Es cierto que esta estrategia evasiva está atestiguada:

$$
\text { (10) /niSkat/ [Øi אkat] 'quijada’ (Sem.J) }
$$

Sin embargo es más común que los hablantes reemplacen la nasal velar por otro sonido. Es así, que lo reemplazan en la mayoría de los casos por una nasal alveolar (11), pero a veces más bien por una nasal bilabial (12) y se encontró un ejemplo de utilización de una oclusiva velar sonora (13):

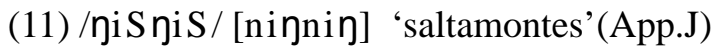

(12) /nuniS/ [muni $\left.\int\right]$ 'barba' (App.D)

(13) /narnarinBa/ [nagarimba] 'verde’ (App.S)

$24 \mathrm{El}$ archifonema /B/ representa los fonemas /b/ y /m/, en variación libre en esta palabra.

$25 \mathrm{El}$ archifonema /S/ representa los fonemas /S/ et / $\mathrm{Q} /$, en variación libre en muchas palabras.

26 C. Grinevald \& al., op. cit. 
Los sonidos de substitución están todos presentes en los inventarios fonológicos de la (las) lengua(s) cotidianamente utilizado(s) por los ramas: el criollo a base inglesa de la región y el español (lengua de la escuela). Además, cada uno tiene al menos uno de dos rasgos características del sonido [ฤ]: [+nasal] o [DORSAL].

\section{Análisis de la pérdida de la nasal velar en función de dos variables}

La variación engendrada por la pérdida de la nasal velar puede ser analizada en función de dos variables: el perfil del locutor y la posición del fonema en la palabra.

El reemplazo del sonido [n] por el sonido [n] así que la supresión son dos estrategias observadas en todos los perfiles de locutores no tradicionales ( [n]: Sem.P, Sem.R, App.J, App.S, App.D ; [Ø] : Sem.J, App.J). Se puede sin embargo observar en los semi-hablantes una preferencia por la supresión, mientras que los principiantes neohablantes prefieren la nasal alveolar.

Por otra parte, las opciones de utilizar [m] o [g] como elementos reemplazantes solo han sido atestiguadas por parte de los principiantes ([m] : App.D, App.S; [g]: App.S). Esas dos realizaciones serían así una evolución más reciente del fenómeno de pérdida de la nasal velar.

Finalmente, la realización [g] es la estrategia menos atestiguada (un solo ejemplo fue recogido de manera indiscutible). Esto se puede explicar por el hecho que este sonido sea bastante raro en la lengua rama ${ }^{27}$.

De manera general, la pérdida de la nasal velar va creciendo según el perfil de los locutores. Así, el fenómeno es inexistente en los locutores tradicionales, débil entre los semi-hablantes y muy avanzado entre los principiantes. Esto refleja de esa forma la progresión de la obsolescencia de la lengua.

A fin de entrever el fenómeno en su totalidad es necesario analizar el lugar ocupado por el fonema en la palabra.

Se puede observar de esa forma que la posición inicial es la más afectada. En efecto, ésa es la única posición en que los semi-hablantes pierden a veces la nasal velar. Por otra parte, y de manera más pronunciada, raros son los ejemplos en que los principiantes neo-hablantes mantienen la nasal velar en posición inicial. En lo que concierne a la posición mediana, las conclusiones son más mitigadas y varían de un locutor al otro. Y finalmente, la posición final está casi totalmente exenta del fenómeno, los neo-locutores principiantes ellos mismos mantienen, en la gran mayoría de los casos, la /n/ en esa posición.

Sabiendo que la nasal velar [ $\eta$ ] es un sonido que puede ocurrir en el criollo del inglés de la región y en el español de Nicaragua ${ }^{28}$, pero solamente en posición final, parece claro que la evolución descrita aquí es debida a la obsolescencia. Dado que los ramas están en situación de bilingüismo -sino de multilingüismo- asimétrico, las características lingüísticas de la(s) lengua(s) dominante(s) tienen fuerte incidencia sobre las de la lengua étnica. Es así que el uso del sonido $/ \mathrm{y} /$ en criollo y en español está suplantando el uso de ese mismo sonido en rama.

La influencia de estas lenguas sobre el rama es tal que aun cuando los locutores están conscientes que deberían de pronunciar [ $\eta$ ] -cuando leen una palabra que contiene el grafema doble "ng" que corresponde a ese sonido en la escritura ordinaria-, generalmente no lo pueden hacer; la competencia de pronunciar este sonido en posición inicial y mediana es frecuentemente casi nula por no haberla adquirido suficientemente temprano en la infancia.

\section{Conclusiones: pérdida de la nasal velar y otros fenómenos fonológicos de obsolescencia en rama}

La pérdida de la nasal velar en posición inicial no es el único fenómeno fonológico de obsolescencia observable en rama. En efecto, hay apócopes vocálicas, degeminaciones y también metástasis, que pueden ser imputadas al estado de ‘lengua en peligro’ en que se encuentra el rama.

Es así que tres realizaciones del lexema /nikiikna/ 'hombre' coexisten.

(14) a. [nikiikna] (Trad.W, Trad.P) / b. [nkiikna] (Trad.P, Miss Nora) / c. [kiikna] (Sem.R, App.D)

La forma completa (14.a) está atestiguada en el habla de los locutores tradicionales. La forma (14b) (apócope) aparece en el habla de los locutores tradicionales y los semi-hablantes. Finalmente, la forma (14.c) (degeminación) está atestiguada en los semi-hablantes y en los neo-hablantes principiantes.

De igual forma, existen tres ${ }^{29}$ maneras de pronunciar / niSniS/ 'saltamontes o chicharra' :

\footnotetext{
27 C. Craig, A Grammar of Rama, op. cit., p. 39.

28 A la diferencia del castellano peninsular.

29 O hasta el doble de esto si se toma en cuenta que /s/ está en variación libre con / S / en esta palabra.
} 
(15) a. [nisnis] (Trad.K, Trad.P, Sem.R, Sem.J) / b. [nisnis] (App.J) / c. [snisni] (App.D)

La forma (15.a) está atestiguada en el habla de los locutores tradicionales y los semi-hablantes. La forma (15b) (nasal velar pronunciada como alveolar) aparece en el habla de los princípiantes. Finalmente, la forma (15.c) (metástasis) fue observada en el habla del principiante D.

Los ejemplos presentados en este artículo nos recuerdan que las lenguas en peligro siguen una evolución diferente de la de las lenguas en plena vitalidad: una evolución acelerada y orientada. Acelerada puesto que las lenguas en peligro son habladas por locutores tradicionales, pero también por locutores no tradicionales, que solo tienen un dominio parcial de la lengua.Y orientada puesto que la situación sociolingüística de los hablantes no tradicionales y el contacto con otras lenguas están directamente al origen de la manera en que los cambios lingüísticos operan.

Adicionalmente hay que notar que estos cambios lingüísticos son complicados por fenómenos de variación. La variación es un hecho intrínseco a las lenguas en peligro, debido a su situación de obsolescencia así como al perfil mayoritario de las comunidades que practican estas lenguas: la mayor parte del tiempo se trata de comunidades poco numerosas. Por eso, los hablantes, acostumbrados a interactuar verbalmente en esa lengua con un número reducido de personas y sin hacer distinciones sociales, no tienen necesidad de adaptar su discurso, lo cual da lugar a una mayor libertad individual y, por consiguiente, a la variación. ${ }^{29}$

\section{REVITALIZACIÓN DEL RAMA}

Al igual que muchos proyectos de revitalización, el proyecto de la lengua rama, aunque atañe a una lengua de tradición oral, se articula alrededor de la puesta en escritura de la lengua. Lo escrito toma así un lugar y un rol preponderante, los cuales van a la par con el contexto de transmisión concebido como dependiente de la escuela.

Esta situación suscita en el momento de la producción de los materiales escolares, no solamente la cuestión del reconocimiento de la variación intrínseca a la lengua, sino también la problemática ligada a quien produce estos materiales y cómo.

\section{Fuentes de variación}

En el seno de los datos ramas puestos a la disposición de la comunidad se pueden observar dos tipos de variación: variaciones internas o externas a la lengua.

Como el formato electrónico facilita la multiplicación de las entradas, las variaciones internas son tomadas en cuenta en el diccionario en línea Turkulka. Ahí se encuentran por ejemplo las variaciones diatópicas $\langle\mathrm{s}\rangle \mid<\mathrm{sh}^{30}>$ (siiru/shiiru 'knife'), o las variaciones diversas (y obsolescencia) de la palabra para "hombre"31.

Además, en el contexto de revitalización de las lenguas en peligro, raramente existe una sola autoridad legítima produciendo la documentación escrita y no todos los que se aventuran a transcribir la lengua están conscientes de sus características fonológicas. Por consiguiente hay que tomar también en consideración las variaciones externas, introducidas por escritos elaborados de manera improvisada, pero que se han convertido en referencias, por tratarse de fuentes escritas (diccionarios, manuales de aprendizaje, etcétera.) $)^{32}$.

\section{Fuentes de variación en los actores de la revitalización}

En 2010, el estudio sociolingüístico conducido por B. Pivot se proponía establecer un diagnóstico de la situación de la revitalización después de veinticinco años de iniciado el proyecto, con el propósito de subrayar qué cambios habían ocurrido a nivel de la comunidad en términos de actitud y práctica lingüística.

La primera constatación es que el proyecto en sí mismo ha ganado la adhesión del conjunto de la comunidad, ahí donde al inicio la pareja Miss Nora-lingüista encontraba, en el mejor de los casos, escepticismo y, en el peor, una franca oposición.

Esto se explica por el trabajo de valorización y el esfuerzo de Miss Nora para transmitir el rama y, en la última década, por el importante rol que ésta ha jugado en el proceso social y político de reconocimiento de los derechos étnicos y territoriales de la comunidad rama.

Este cambio es significativo, porque ha traído al frente del escenario a los hablantes tradicionales y a los semi-hablantes,

29 N. Dorian, Investigating variation: the effects of social organization and social setting, New York : Oxford University Press, 2010.

$30 \quad / \int 1$

$31 \quad$ Ver el ejemplo (14)

32 Ver C. Craig, « Review of N. Rigby \& R. Schneider: Dictionary of the Rama Language: Rama-English- Rama-Creole-Spanish/English-Rama, Speaking with the Tiger, Vol 2 », dans The International Journal of American Linguistics 56/2, Berlin : Dietrich Reimer Verlag, 1990. 
que antes no se atrevían a mostrarse y que no hablaban en público su lengua. Ahora, ellos constituyen el Consejo de hablantes, grupo formal mixto compuesto de hablantes tradicionales, semi-hablantes, neo-hablantes y principiantes, que representan el 'saber lingüístico' y que son consultados, por ejemplo, para la creación de nuevas palabras.

Además de esta nueva situación, la actitud positiva y el apoyo de parte de la comunidad ha legitimado el involucramiento de nuevos actores en el proceso de revitalización, aunque no hablen muy bien el idioma: los transmisores (hablantes principiantes). Estos han aprendido el rama esencialmente en los documentos producidos por el Proyecto de la lengua Rama, y por consiguiente tienen un saber principalmente escrito. El contexto sociolingüístico, al igual que los errores presentes en ciertos documentos producidos por el Proyecto, introducen cambios lingüísticos radicales. Por consiguiente, la lengua que enseñan es necesariamente una variedad marcada por la obsolescencia.

Finalmente, la revitalización ha dado nacimiento a neohablantes, hablantes del rama del futuro. Su dominio limitado de la lengua, reforzado por las pocas ocasiones que tienen de practicarla y la poca presión social ${ }^{33}$, hará que los que decidirán hablar rama a sus hijos transmitirán seguramente variantes obsolescentes.

\section{Repensar la revitalización, repensar la variación}

La revitalización es una dinámica que se articula alrededor de una comunidad, para responder a necesidades que son las suyas y a las cuales el lingüista debe adaptarse.

Así, cómo se ha visto en otros $\operatorname{casos}^{34}$, el estudio de 2010 mostró que la comunidad rama no buscaba en su proyecto de revitalización la "re-vernaculizacion” de su lengua. En efecto, el valor atribuido no es el que corresponde a la comunicación conversacional sino un valor simbólico que permite a los ramas identificarse y hacerse identificar en tanto que etnia legítima en la jerarquía social y sobre su territorio. Por consiguiente, no les es necesario hablar el rama de manera fluida.

Los programas de revitalización deberían de abordar más la lengua como acto de palabra que como producción normada y considerar que se ha logrado el objetivo cuando las partes involucradas están satisfechas, en vez de considerar la variación como una violación de la 'norma'.

La cuestión que se plantea entonces es: ¿cómo tomar en cuenta la variación para la revitalización?
Para un lingüista, esta pregunta puede aparecer al momento de elaborar un diccionario: ¿Qué forma privilegiar en las entradas léxicas?

Para un actor de la revitalización, la escogencia de la variante a privilegiar para la producción de los documentos de transmisión y de valorización puede ser conflictual y hacerse sobre un fondo de luchas ideológicas donde se afrontan corrientes diversas, o puede también resultar de un cierto consenso.

Para la comunidad lingüística se va a tratar sobre todo de una cuestión de aceptación y de reconocimiento de la variación como forma legitimada por el uso. Esto dependerá en parte del valor de esta lengua para la comunidad al igual que también del número de hablantes y de la difusión de los documentos que atestigüen una 'norma' establecida en un instante $\mathrm{T}$ de la historia de la descripción y transcripción de esa lengua.

\section{CONCLUSIÓN}

Este estudio presentó un caso de cambio lingüístico en el contexto de una lengua en peligro. Se escogió aquí concentrar la atención sobre la fonología, para ilustrar los cambios atribuibles a la obsolescencia, detallando la pérdida progresiva de la nasal velar en rama.

Este ejemplo ha permitido poner en relieve dos factores: los nuevos perfiles de hablantes y el fenómeno de contacto de lenguas.

Los cambios debidos a la obsolescencia son interesantes en el sentido que pueden ser estudiados fácil y directamente y que intervienen en un contexto donde la variación está ya fuertemente presente.

La consideración de estos cambios implica la necesidad absoluta de repensar la noción de revitalización al igual que nuestro comportamiento de cara a la variación por una parte y a la evolución de las lenguas por otra.

Toda lengua se inscribe en un proceso natural de evolución, de modificaciones, que incluso para las lenguas estandarizadas terminan por ser integradas en la norma. La situación particular de las lenguas en peligro es que estos cambios se hacen, en el uso oral, extremadamente rápido, creando una fuerte variación entre los hablantes en un momento dado. El reto de la revitalización es conseguir integrar las variantes a la norma escrita y oral emergentes, cuando, en los contextos de las lenguas estandarizadas, resultan tan difíciles de aceptar.

33 Dos parámetros que normalmente encuadran y aseguran una cierta norma lingüística (N. Dorian, Investigating variation, op. cit.).

34 D. Cameron, « Language Endangerment and Verbal Hygiene: History, Morality and Politics » dans A. Duchêne \& M.Heller (éd.), Discourses of endangerment, Londres/New-York : Continuum, 2007 ; A. Jaffre, « Parlers et idéologieslangagières », Ethnologie française 38/3, 2008. 


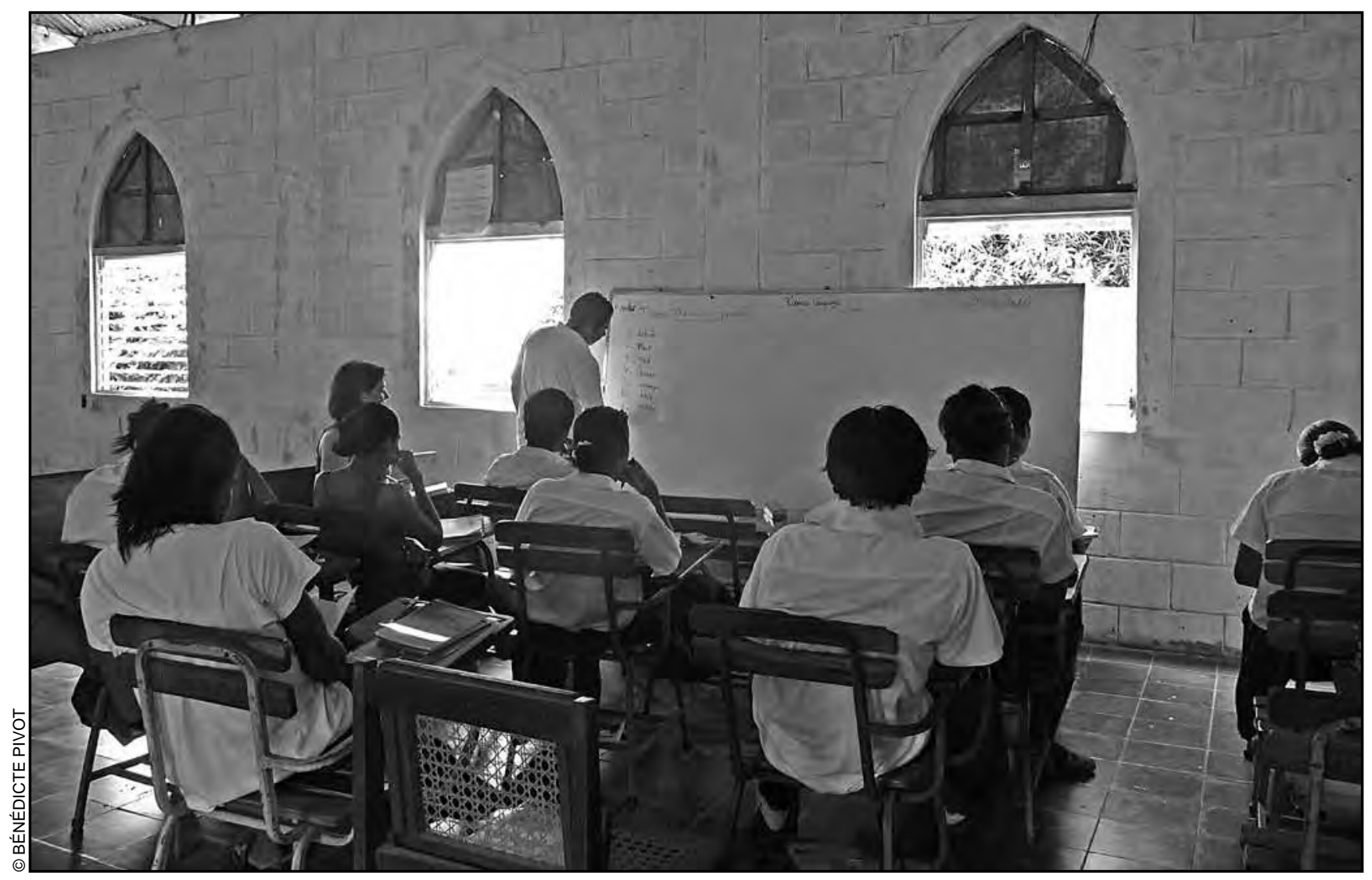

Clase de Rama en RAMAKI, Marzo 2010.

\section{Referencias.}

Bert M., Grinevald C., « Proposition de typologie des locuteurs de LED », en C. Grinevald \& M. Bert (dir.), Linguistique de terrain sur langues en danger, Faits de langues 35-36, Paris : Ophrys, 2010, p. 125-126.

Cameron D., « Language Endangerment and Verbal Hygiene: History, Morality and Politics » en A. Duchêne \& M. Heller (éd.), Discourses of endangerment, Londres/New-York : Continuum, 2007, p. 268-285.

Campbell L., Muntzel M. C., « The structural consequences of language death », dans N. Dorian (éd.), Investigating obsolescence. Studies in language contraction and death, Cambridge : Cambridge

University Press, 1989, p. 181-196.

Chevrier N., Evolution phonologique d'une langue en danger : le cas du rama du Nicaragua, Université Lyon 2 : mémoire de master 2 : Sciences Du Langage, 2011.

Costa J., « Des derniers locuteurs aux néo-locuteurs : revitalisation linguistique en Europe », dans C. Grinevald \& M. Bert (dir.), Linguistique de terrain sur langues en danger, Faits de langues 35-36,

Paris : Ophrys, 2010. p. 205-224.

Craig C., " A constitutional response to language endangerment: the case of Nicaragua », Language 68/1, 1992, p. 17-24. 
Craig C., " Language Contact and Language Degeneration », dans F. Coulmas (éd.), The Handbook of Sociolinguistics, Oxford : Blackwell, 1997, p. 257-270.

Craig C., « Language Shift and Language Death: The case of Rama in Nicaragua », International Journal of the Sociology of Language 93, Berlin/New-York : Mouton de Gruyter, 1993, p. 11-26.

Craig C., « Review of N. Rigby \& R. Schneider: Dictionary of the Rama Language: Rama-English-RamaCreole-Spanish/English-Rama, Speaking with the Tiger, Vol 2 », en The International Journal of American Linguistics 56/2, Berlin : Dietrich Reimer Verlag, 1990, p. 293-304.

Craig C., A Grammar of Rama (Report to National Science Foundation BNS 8511156), 1988.

Dorian N. (éd.), Investigating obsolescence. Studies in language contraction and death, Cambridge : Cambridge university Press, 1989.

Dorian N., Investigating variation: the effects of social organization and social setting, New York : Oxford University Press, 2010.

Grinevald C., « Linguistique de terrain sur deux langues en danger : locuteurs et méthodes », en C. Grinevald \& M. Bert (dir.), Linguistique de terrain sur langues en danger, Faits de langues 35-36,

Paris : Ophrys, 2010, p. 133-177.

Grinevald C., «Quarante ans de perspective sur deux langues en danger : le jakaltek popti’ du Guatemala et le rama du Nicaragua », en C. Grinevald \& M. Bert (dir.), Linguistique de terrain sur

langues en danger, Faits de langues 35-36, Paris : Ophrys, 2010, p. 39-78.

Grinevald C., « Why the Tiger language and not Rama Cay Creole? Language revitalization made harder », en P. K. Austin (éd.), Language Documentation and Description 3, London : HRELP SOAS, 2005, p. 196-224.

Grinevald C., Costa J., « Langues en danger : le phénomène et la réponse des linguistes », en C. Grinevald \& M. Bert (dir.), Linguistique de terrain sur langues en danger, Faits de langues 35-36,

Paris : Ophrys, 2010, p. 23-37.

Grinevald C., Soubrier A., Assadi B., Kauffmann M., Torres C. \& Pivot B., Turkulka: diccionario de lengua rama,2002-2006. En línea: <www.turkulka.net>

Holm J. A., « Nicaragua’s Miskito Coast Creole English », dans J. A. Holm (éd.), Central American English. Varieties of English Around the World vol. 2, Heidelberg : J.Groos, 1983, p. 95-130.

Jaffre A., « Parlers et idéologies langagières », Ethnologie française 38/3, 2008, p. 517-526.

Moseley C. (éd.), Atlas des langues en danger dans le monde 3e édition, Paris : Editions UNESCO, 2010. Version en línea: <http://www.unesco.org/culture/en/endangeredlanguages/atlas>

Pivot B., Evaluation d’une situation d'une langue en danger, le cas du rama au Nicaragua, Université Lyon 2 : mémoire de master 2 : Sciences Du Langage, 2010.

Riverstone G., La tierra de nuestros ancestros. Territorio Rama y Creole en el Caribe Nicaragüense, Managua, Nicaragua : IBIS, 2008. 